\title{
Ergonomic Improvement of Drinking Water Dispenser for Indonesian People Using Rapid Upper Limb Assessment Method
}

\author{
F.A. Bayu Satya Wijaya ${ }^{1}$, Bethriza Hanum ${ }^{2}$ and Hardi Triyono Putra \\ ${ }^{1-3}$ Industrial Engineering Department \\ Faculty of Engineering \\ University of Mercu Buana \\ Jl. Raya Meruya Selatan, Kembangan, Jakarta 11650
}

Indonesia

\begin{abstract}
The existing water dispenser has been established in Indonesia for years which has various water tap designs and height levels as well. Unfortunately, the existing water tap design and height level have suited only for certain people with a height of less than $167 \mathrm{~cm}$ and therefore other taller people experienced bending posture when operating it. Furthermore, the water tap valve which required both hands to operate has made another effort to fill into the water media. Several research and methodologies have been proposed regarding water tap such as how to avoid water spills using the TRIZ method and how to enhance the smart dispenser through the Internet of Time. None of it discovered how to enhance its ergonomic aspect. However, to achieve improve the ergonomic level, several experiments and measurements have been performed in this research using Rapid Upper Limb Assessment (RULA) method, to generate a recommendation for ergonomic water dispensers.
\end{abstract}

Keywords: Ergonomic, Water Dispenser, Water Tap Valve, RULA.

\section{INTRODUCTION}

Human body requires water for the active and healthy life. Water requirements can vary depends on physical activity, age groups, body weight, climate, and diet (energy intake). According to Irianto (2017), water is the biggest component of human body which contribute $60 \%-70 \%$ of body weight and therefore water is needed especially during sport activity or other heavy activity. A human requires 2.5 liter of water or equal to eight glass on daily basis (Asmadi et all, 2011). The awareness to drink at proper volume of water has become common recently by keeping it into water dispenser or other drinking media.

Water dispenser has been established in Indonesia for years. It has various water tap valve design and height level. To operate water tap valve, it has to be press or push by either single or both hands. Current water tap height level varies from $77 \mathrm{~cm}$ to 81 $\mathrm{cm}$. Unfortunately particular users have experienced uncomfortable (bending) posture when tapping down the water from a dispenser, as shown in the following figure.
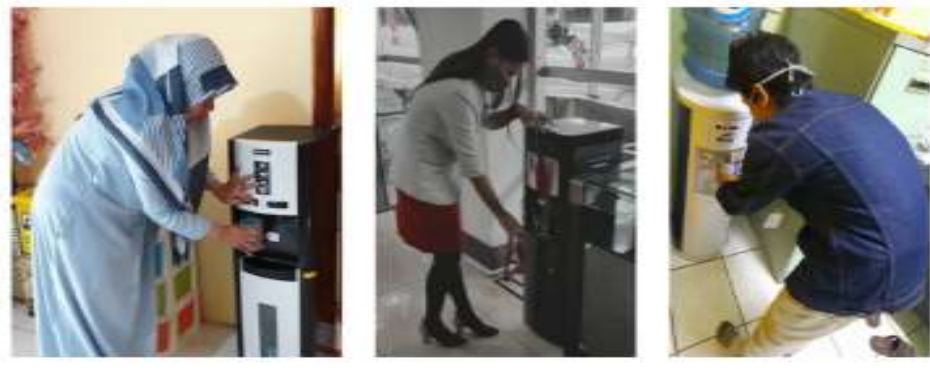

Figure 1 Body Posture during Water Dispenser Operation 
A new standard and measurement need to be carried out to recommend more ergonomic posture during operate a dispenser. Several research and methodologies have been performed regarding to water tap such as how to avoid water spill using TRIZ method and how to enhance a smart water dispenser using Internet of Things, but none studied on how to improve ergonomic aspect of water dispenser.

Following to mentioned situation above, this research offered an improvement from ergonomic aspect of a water dispenser design using Rapid Upper Limb Assessment (RULA) method. The respondent data has randomly selected from respected adult users in Jakarta , Indonesia. User's habit in using water dispenser has been documented, measured and analyzed to recommend proposed improvement. Mc Attamney and Corlett (1993) proved that RULA method can investigate for work related upper limb disorders.

This paper addresses the un-comfort (bending) body posture using RULA method. The rest of this paper is organized as follows : first, a brief description of water dispenser is provided. Then, user's measurement and analysis are described, followed by recommended water dispenser design to improve ergonomic level. Finally, the paper is concluded.

\section{LITERATURE SURVEY}

\subsection{The Rapid Upper Limb Assessment}

Dr Lynn McAtamney and Professor E. Nigel Corlett developed the Rapid Upper Limb Assessment (RULA) method as a postural targeting method for estimating the risks of work-related upper limb disorders. A RULA assessment gives a quick and systematic assessment of the postural risks to a worker. The analysis can be accomplished before and after an intervention. This is to demonstrate that the intervention has worked to lower the risk of injury.

RULA method uses diagram or picture of body posture and three tables to evaluate risk factors. McPhee (1987) stated that risk factor for external loads factors are quantity of motion, static muscular work, force, working posture and restless working time. The stepping assessment and scoring sheet are shown in the following figure.

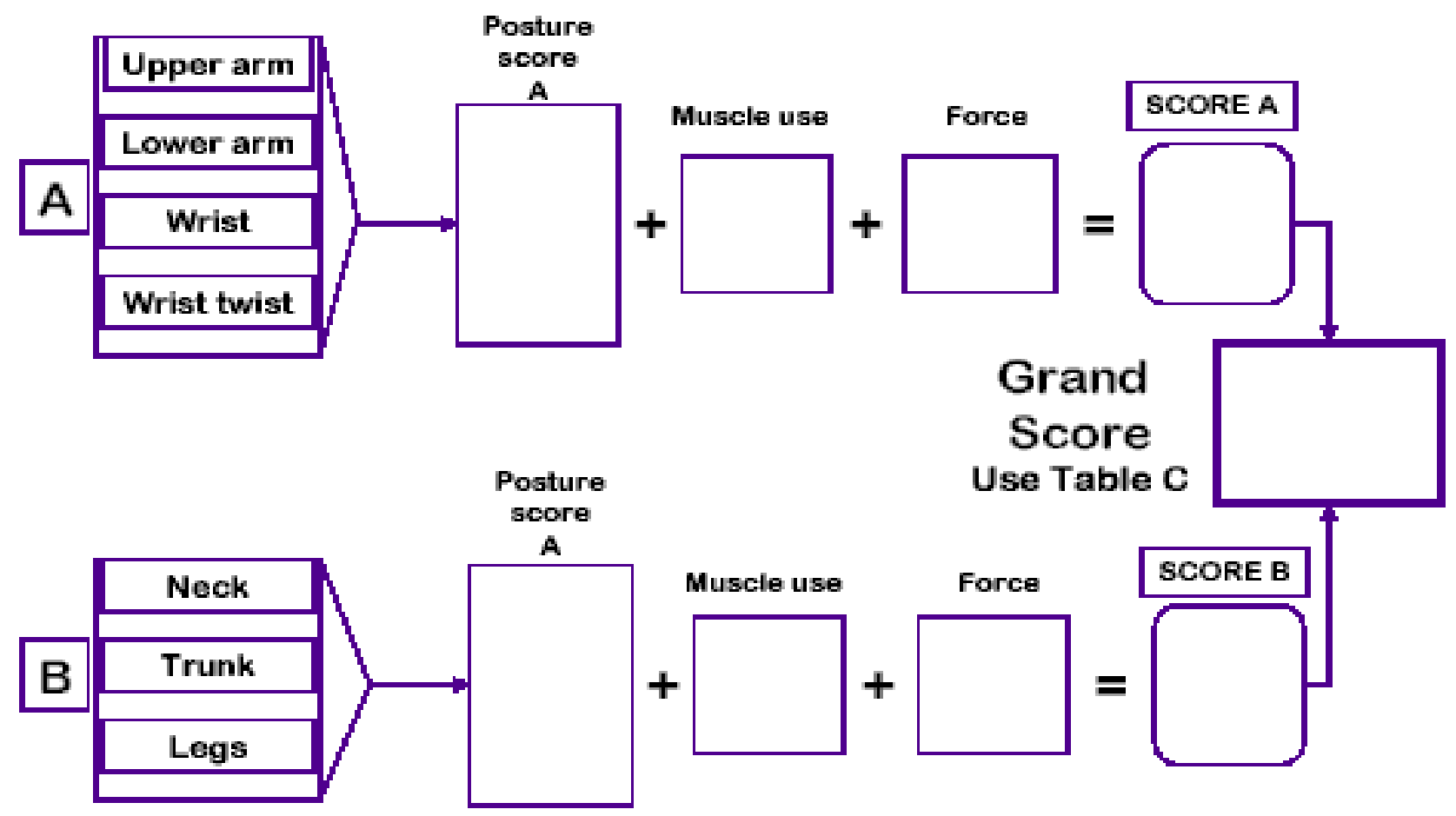

Figure 2. RULA Score Sheet

The Rapid Upper Limb Assessment (RULA) action levels gives the urgency about the need to change how a person is working. It works as a function of the degree of injury risk.

1. Action Level 1 - RULA score 1-2 means that the person is working in the best posture. There is no risk of injury from their work posture. 
2. Action Level 2-RULA score 3-4 means that the person is working in a posture that could present some risk of injury from their work posture. This score most likely is the result of one part of the body being in a deviated and awkward position. Investigate the reasons and correct.

3. Action Level 3 - RULA score 5-6 means that the person is working in a poor posture with a risk of injury from their work posture. Investigate the reasons and change them in the near future to prevent an injury.

4. Action Level 4 - RULA score 7-8 means that the person is working in the worst posture with an immediate risk of injury from their work posture. Investigate the reasons and change them in the near future to prevent an injury.

\section{OBJECTIVE OF THE RESEARCH}

The objective of the current research effort is to improve ergonomic aspect of water dispenser by further focusing on how to

1. Define proper water tap height level for Indonesian people

2. Define comfort water tap valve mechanism

\subsection{RESEARCH METHODOLOGY}

RULA method has been mainly used to assess upper limb disorder. Several methodologies have also been applied such as Nordic Body Map assessment and anthropometric measurement approach for related human body part. As overall, the research effort has been performed by carried out these following stages as shown in this figure below.

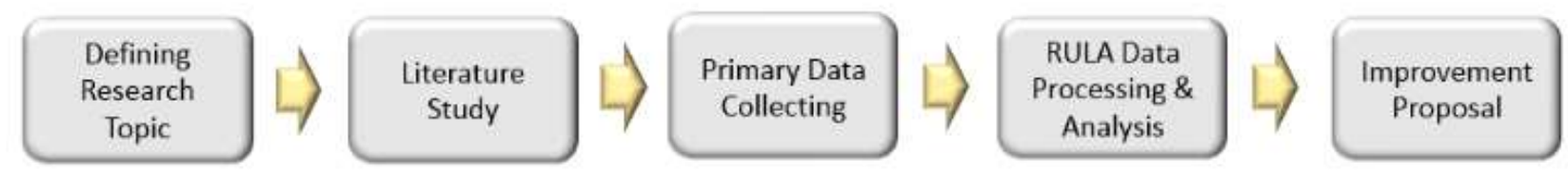

Figure 3. Research Stages

First, research topic defined and carried out literature study. It then followed by primary data collecting for several data such as user's documentation when using water dispenser naturally, user habit investigation, anthropometric measurement and Nordic Body Map. Then it followed by RULA data processing and analysis by assessing RULA scoring sheet to investigate further improvement proposal needed.

\section{RESULT AND DISCUSSION}

4.1 User Habit, Nordic Body Map Result and Anthropometric Measurement

According to 50 user interview during data collecting activity, several drinking pattern as shown in the table 1 .

Table 1. Activity and Water Consumption Pattern

\begin{tabular}{|c|c|c|c|}
\hline Activity Pattern & Quantity & $\%$ & Water Consumption \\
\hline $\begin{array}{l}\text { 1. } \text { Pattern 1: } \\
\text { - At Home, } 15 \text { hours } \\
\text { - At Working Place, } 9 \text { hours }\end{array}$ & 36 resp. & 72 & $\begin{array}{ll}\text { - } & 1200 \mathrm{ml} \\
\text { - } & 1500 \mathrm{ml}\end{array}$ \\
\hline $\begin{array}{l}\text { 2. Pattern 2: } \\
\text { - At Home, } 14 \text { hours } \\
\text { - At Working Place, } 10 \text { hours }\end{array}$ & 9 resp. & 18 & $\begin{array}{ll}\text { - } & 1000 \mathrm{ml} \\
\text { - } & 1600 \mathrm{ml}\end{array}$ \\
\hline $\begin{array}{l}\text { 3. } \text { Pattern } 3 \text { : } \\
\text { - At Home, } 13 \text { hours } \\
\text { - At Working Place, } 11 \text { hours }\end{array}$ & 5 resp. & 10 & $\begin{array}{ll}\text { - } & 1000 \mathrm{ml} \\
\text { - } & 1500 \mathrm{ml}\end{array}$ \\
\hline Total & 50 resp. & 100 & \\
\hline
\end{tabular}


User's pattern to consume water was spread at two places and all of them consumed it at higher water volume at the office or other working place. Every user has its own preference to use drinking media such as a mug or glass with $300 \mathrm{ml}$ to $500 \mathrm{ml}$ capacity and 1 liter of drinking bottle. Each media has its own tapping duration and gross weight during the usage which shown in table as follows.

Table 2. Water Media Usage Data

\begin{tabular}{|c|c|c|c|c|}
\hline Water Media Size & Tapping Duration & Gross Weight & User Preference & $\%$ \\
\hline $300 \mathrm{ml}$ & $15 \mathrm{sec}$ & $0,6 \mathrm{~kg}$ & 11 resp. & 22 \\
\hline $500 \mathrm{ml}$ & $25 \mathrm{sec}$ & $0,65 \mathrm{~kg}$ & 35 resp. & 70 \\
\hline $1000 \mathrm{ml}$ & $50 \mathrm{sec}$ & $1,15 \mathrm{~kg}$ & 4 resp. & 8 \\
\hline \multicolumn{3}{|r|}{ Total } & 50 resp. & \\
\hline
\end{tabular}

Most of users preferred to use $500 \mathrm{ml}$ water media due to proper volume, tapping time and gross weight to be handled during operate a water dispenser. At the office, the users had to refill the media at least three times a day. On the other side, this research has also documented the type of water dispenser that frequently used by the users to investigate further aspect which may affect potential upper limb disorder, as shown in the following table.

Table 3. Water Dispenser Type and Usage

\begin{tabular}{|c|c|c|c|}
\hline Water Dispenser & Type A & Type B & Type C \\
\hline & & \\
Figure & & & \\
& & & \\
& & & \\
\hline Water Tap Height & $77 \mathrm{~cm}$ & $80 \mathrm{~cm}$ & $81 \mathrm{~cm}$ \\
\hline Dispenser Usage & 7 & 31 & 12 \\
\hline Percentage & $14 \%$ & $62 \%$ & $24 \%$ \\
\hline
\end{tabular}

Almost $90 \%$ of users used two type of water dispenser B and C both at home and at the office, then it has been used as main focus in further observation. The height level of water tap is higher than type A which enable to enhance ergonomic posture during water dispenser operation. In fact, these following figure has shown the body posture of some water dispenser's user.

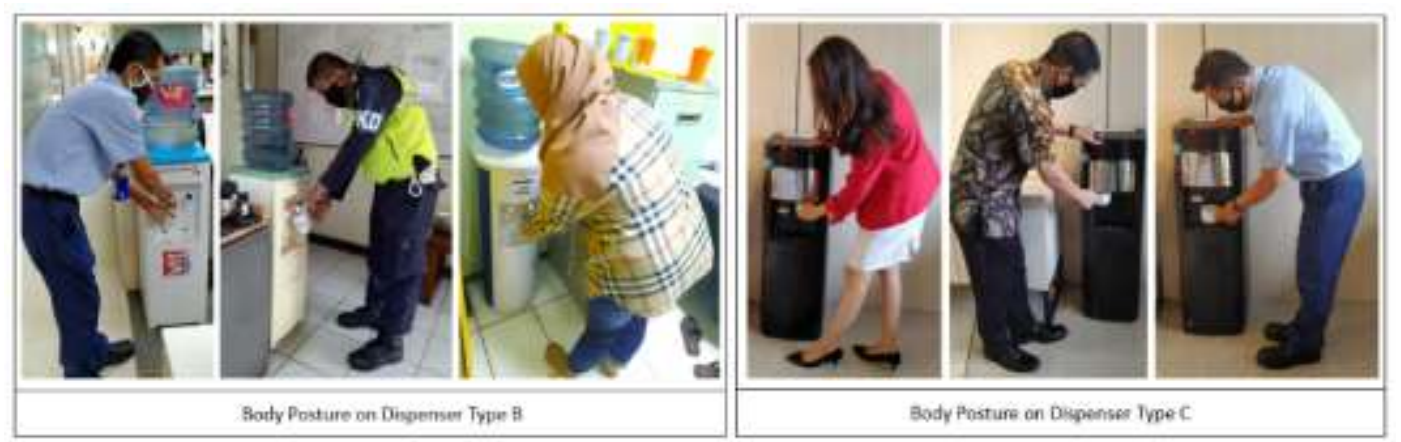

Figure 4. Body Posture on Dispenser Type B and Type C

The body posture has bended around $20^{\circ}$ to $30^{\circ}$ as well as the neck at various angle for around less than 1 minute depending on the media used and the expected water level. Upper arm and lower had also to open at certain degree to fit water tap height level. Anyway, all user had also to use both hands to operate water tap valve on a dispenser. Each hand has its role to grab the glass or 
other water media and to push tap button or valve. These following table has shown various water tap valve type used in this research.

Table 4. Water Tap Valve Type

\begin{tabular}{|c|c|c|c|}
\hline Water Tap Valve & Type A & Type B & Type C \\
\hline Figure & $\begin{array}{c}\text { Single hand, push the } \\
\text { water valve by water } \\
\text { media. Other hand is } \\
\text { free }\end{array}$ & $\begin{array}{c}\text { Double hand, one to } \\
\text { press the water valve, } \\
\text { other to grab water } \\
\text { media at the same } \\
\text { height level }\end{array}$ & $\begin{array}{c}\text { Double hand, one to } \\
\text { press the water valve, } \\
\text { other to grab water } \\
\text { media at different } \\
\text { height level }\end{array}$ \\
\hline Dispenser Usage & 10 & 28 & 12 \\
\hline Percentage & $20 \%$ & $56 \%$ & $24 \%$ \\
\hline
\end{tabular}

According to usage description above, water tap valve type A basically may provide better comfort for user rather than other two types since there is only single hand used to operate water tap valve though there are two roles of the hand that is to grab the water media and to push the water valve simultaneously.

This research recorded the Nordic Body Map measurement as initial reference to investigate any complaint of Musculoskeletal Disorders (MSDs) for each user, which is shown in this following table.

Table 5. The Nordic Body Map Evaluation

\begin{tabular}{|c|l|c|c|c|c|}
\hline \multicolumn{1}{|c|}{ Location } & A & B & C & D \\
\hline 0 & Pain in the upper neck & $12 \%$ & $66 \%$ & $22 \%$ & - \\
\hline 1 & Pain in the lower neck & $48 \%$ & $52 \%$ & - & - \\
\hline 2 & Pain in the left shoulder & $78 \%$ & $22 \%$ & - & - \\
\hline 3 & Pain in the right shoulder & $12 \%$ & $88 \%$ & - & - \\
\hline 4 & Pain in the left upper arm & $12 \%$ & $88 \%$ & - & - \\
\hline 5 & Pain in the back & $12 \%$ & $66 \%$ & $22 \%$ & - \\
\hline 6 & Pain in the right upper arm & $12 \%$ & $88 \%$ & - & - \\
\hline 7 & Pain in the waist & $12 \%$ & $58 \%$ & $30 \%$ & - \\
\hline 8 & Pain in the buttock & $12 \%$ & $66 \%$ & $22 \%$ & - \\
\hline 13 & Pain in the right lower arm & $12 \%$ & $88 \%$ & - & - \\
\hline 15 & Pain in the right wrist & $12 \%$ & $88 \%$ & - & - \\
\hline 17 & Pain in the right hand & $12 \%$ & $88 \%$ & - & - \\
\hline
\end{tabular}

The table above has described that using water dispenser has raised potential MSDs on the upper limb of the body 2 especially around the neck, right shoulder, around the upper arm, in the back, waist to buttock and around lower arm as well as around right wrist and right hand. For some users who has height more than $167 \mathrm{~cm}$, the pain tend to appear around upper neck, in the back, in the waist and also in the buttock.

For ergonomic improvement purposes, the respected anthropometric data has been measured as a main reference in redesigning particular part or water dispenser, especially related the water tap. The table as shown as follows.

Table 6. Anthropometric Data

\begin{tabular}{|c|c|c|}
\hline Category & Average & Percentile 95 \\
\hline Body Height & $166.12 \mathrm{~cm}$ & $174 \mathrm{~cm}$ \\
\hline Elbow Height & $98.88 \mathrm{~cm}$ & $103.55 \mathrm{~cm}$ \\
\hline Lower Arm Length & $30.13 \mathrm{~cm}$ & $30.50 \mathrm{~cm}$ \\
\hline
\end{tabular}


International Journal of Engineering Research And Advanced Technology, Vol.6, Issue 8, August-2020

\subsection{Rapid Upper Limb Assessment (RULA) Result}

Following to RULA method, each angle of user's body posture has been measured by using two groups classification A and B.

The detail assessment as shown in this following table.

Table 7. RULA Result

\begin{tabular}{|c|c|c|c|c|c|c|c|c|c|c|c|c|c|}
\hline \multirow{2}{*}{ No } & \multirow{2}{*}{ Height } & \multirow{2}{*}{$\begin{array}{l}\text { Elbow } \\
\text { Height }\end{array}$} & \multirow{2}{*}{$\begin{array}{l}\text { Lower } \\
\text { Arm } \\
\text { Length }\end{array}$} & $\begin{array}{c}\text { Group A } \\
\text { (Arm) }\end{array}$ & $\begin{array}{l}\text { 1. Upper } \\
\text { Arm }\end{array}$ & $\begin{array}{l}\text { 2. Lower } \\
\text { Arm }\end{array}$ & $\begin{array}{l}\text { 3. Wrist } \\
\text { Position }\end{array}$ & $\begin{array}{l}\text { 4. Wrist } \\
\text { Twist }\end{array}$ & \multirow{2}{*}{$\begin{array}{l}\text { Table } \\
\text { Score }\end{array}$} & \multirow{2}{*}{ Activity } & \multirow{2}{*}{ Load } & \multirow{2}{*}{ Score } & \multirow{2}{*}{$\begin{array}{l}\text { Final } \\
\text { Score }\end{array}$} \\
\hline & & & & $\begin{array}{c}\text { Group B } \\
\text { (Body) }\end{array}$ & 1. Neck & 2. Trunk & 3. Leg & - & & & & & \\
\hline \multirow{2}{*}{1} & \multirow{2}{*}{157} & \multirow{2}{*}{96} & \multirow{2}{*}{30} & $\mathrm{~A}$ & 2 & 1 & 2 & 2 & 3 & 0 & 1 & 4 & \multirow{2}{*}{3} \\
\hline & & & & B & 2 & 2 & 1 & - & 2 & 0 & 1 & 3 & \\
\hline \multirow{2}{*}{2} & \multirow{2}{*}{160} & \multirow{2}{*}{96.5} & \multirow{2}{*}{30} & A & 2 & 1 & 3 & 2 & 4 & 0 & 1 & 5 & 4 \\
\hline & & & & $\mathrm{B}$ & 2 & 2 & 1 & - & 2 & 0 & 1 & 3 & 4 \\
\hline & & & & A & 1 & 1 & 1 & 2 & 2 & 0 & 1 & 3 & 3 \\
\hline 3 & 165 & 97.5 & 30 & B & 2 & 2 & 1 & - & 2 & 0 & 1 & 3 & 3 \\
\hline & & & & $\mathrm{A}$ & 2 & 1 & 2 & 2 & 3 & 0 & 1 & 4 & 3 \\
\hline 4 & 164 & 91 & 30 & B & 2 & 2 & 1 & - & 2 & 0 & 1 & 3 & 3 \\
\hline 5 & 164 & 07 & 30 & $\bar{A}$ & 2 & 1 & 2 & 2 & 3 & 0 & 1 & 4 & 3 \\
\hline 5 & 164 & 91 & 30 & B & 2 & 2 & 1 & - & 2 & 0 & 1 & 3 & 3 \\
\hline 6 & & 075 & 30 & $\mathrm{~A}$ & 2 & 1 & 2 & 2 & 3 & 0 & 1 & 4 & 4 \\
\hline 6 & 163 & 91.5 & 30 & B & 3 & 2 & 1 & - & 3 & 0 & 1 & 4 & 4 \\
\hline 7 & & 085 & & $\mathrm{~A}$ & 2 & 1 & 2 & 2 & 3 & 0 & 1 & 4 & 4 \\
\hline 1 & $16 /$ & 98.5 & 30.5 & B & 3 & 2 & 1 & - & 3 & 0 & 1 & 4 & 4 \\
\hline & & & & A & 2 & 1 & 2 & 2 & 3 & 0 & 1 & 4 & 5 \\
\hline 8 & 167 & 98.5 & 30.5 & B & 2 & 3 & 1 & - & 4 & 0 & 1 & 5 & 5 \\
\hline 0 & & 08 & 30 & $\mathrm{~A}$ & 2 & 1 & 3 & 2 & 4 & 0 & 1 & 5 & 6 \\
\hline 9 & 165 & 98 & 30 & B & 2 & 3 & 1 & - & 4 & 0 & 1 & 5 & 6 \\
\hline & & & & $\mathrm{A}$ & 2 & 1 & 2 & 2 & 3 & 0 & 1 & 4 & 4 \\
\hline 10 & 167 & 98.5 & 30.5 & B & 3 & 2 & 1 & - & 3 & 0 & 1 & 4 & 4 \\
\hline & & & & $\mathrm{A}$ & 1 & 1 & 1 & 2 & 2 & 0 & 1 & 3 & \\
\hline 11 & 158 & 96 & 30 & B & 3 & 2 & 1 & - & 3 & 0 & 1 & 4 & 4 \\
\hline & & & & $\mathrm{A}$ & 2 & 1 & 2 & 2 & 3 & 0 & 1 & 4 & 3 \\
\hline 12 & 162 & 97 & 30 & B & 2 & 2 & 1 & - & 2 & 0 & 1 & 3 & 3 \\
\hline 12 & 162 & 075 & 30 & $\mathrm{~A}$ & 2 & 1 & 3 & 2 & 4 & 0 & 1 & 5 & 4 \\
\hline 13 & 163 & 91.5 & 30 & B & 2 & 2 & 1 & - & 2 & 0 & 1 & 3 & 4 \\
\hline 14 & 166 & 085 & 30 & $\mathrm{~A}$ & 2 & 1 & 2 & 2 & 3 & 0 & 1 & 4 & 4 \\
\hline 14 & 166 & 98.5 & 30 & B & 3 & 2 & 1 & - & 3 & 0 & 1 & 4 & 4 \\
\hline & & & 305 & $\mathrm{~A}$ & 2 & 1 & 3 & 2 & 4 & 0 & 1 & 5 & \\
\hline 15 & $1 / 4$ & 103 & 30.5 & B & 2 & 3 & 1 & - & 4 & 0 & 1 & 5 & 6 \\
\hline 16 & 167 & 08 & 305 & $\mathrm{~A}$ & 2 & 1 & 2 & 2 & 3 & 0 & 1 & 4 & 3 \\
\hline 16 & 167 & 98 & 30.5 & B & 2 & 2 & 1 & - & 2 & 0 & 1 & 3 & 3 \\
\hline & & & & $\mathrm{A}$ & 2 & 1 & 3 & 2 & 4 & 0 & 1 & 5 & 5 \\
\hline 17 & 165 & 97.5 & 30 & B & 3 & 2 & 1 & - & 3 & 0 & 1 & 4 & 5 \\
\hline & 167 & 095 & & $\mathrm{~A}$ & 2 & 1 & 2 & 2 & 3 & 0 & 1 & 4 & \\
\hline 18 & 167 & 98.5 & 30 & B & 2 & 2 & 1 & - & 2 & 0 & 1 & 3 & 3 \\
\hline & & & & $\mathrm{A}$ & 2 & 1 & 2 & 2 & 3 & 0 & 1 & 4 & \\
\hline 19 & 164 & 97 & 30 & B & 2 & 2 & 1 & - & 2 & 0 & 1 & 3 & 3 \\
\hline 20 & 160 & 005 & 305 & $\mathrm{~A}$ & 2 & 1 & 2 & 2 & 3 & 0 & 1 & 4 & 3 \\
\hline 20 & 169 & 99.5 & 30.5 & B & 2 & 2 & 1 & - & 2 & 0 & 1 & 3 & 3 \\
\hline 21 & 167 & 085 & 30 & $\mathrm{~A}$ & 1 & 1 & 1 & 2 & 2 & 0 & 1 & 3 & \\
\hline 21 & 167 & 98.5 & 30 & B & 2 & 2 & 1 & - & 2 & 0 & 1 & 3 & 3 \\
\hline 22 & 165 & 075 & 30 & $\mathrm{~A}$ & 2 & 1 & 2 & 2 & 3 & 0 & 1 & 4 & 3 \\
\hline 22 & 165 & 97.5 & 30 & B & 2 & 2 & 1 & - & 2 & 0 & 1 & 3 & 3 \\
\hline & & & & $\mathrm{A}$ & 2 & 1 & 2 & 2 & 3 & 0 & 1 & 4 & 4 \\
\hline 23 & 166 & 98 & 30 & B & 3 & 2 & 1 & - & 3 & 0 & 1 & 4 & 4 \\
\hline
\end{tabular}


International Journal of Engineering Research And Advanced Technology, Vol.6, Issue 8, August-2020

Table 7. RULA Result (Continued)

\begin{tabular}{|c|c|c|c|c|c|c|c|c|c|c|c|c|c|}
\hline \multirow{2}{*}{ No } & \multirow{2}{*}{ Height } & \multirow{2}{*}{$\begin{array}{l}\text { Elbow } \\
\text { Height }\end{array}$} & \multirow{2}{*}{$\begin{array}{l}\text { Lower } \\
\text { Arm } \\
\text { Length }\end{array}$} & $\begin{array}{c}\text { Group A } \\
\text { (Arm) }\end{array}$ & $\begin{array}{l}\text { 3. Upper } \\
\text { Arm }\end{array}$ & $\begin{array}{l}\text { 4. Lower } \\
\text { Arm }\end{array}$ & $\begin{array}{l}\text { 3. Wrist } \\
\text { Position }\end{array}$ & $\begin{array}{l}\text { 4. Wrist } \\
\text { Twist }\end{array}$ & \multirow{2}{*}{$\begin{array}{l}\text { Table } \\
\text { Score }\end{array}$} & \multirow{2}{*}{ Activity } & \multirow{2}{*}{ Load } & \multirow{2}{*}{ Score } & \multirow{2}{*}{$\begin{array}{l}\text { Final } \\
\text { Score }\end{array}$} \\
\hline & & & & $\begin{array}{c}\text { Group B } \\
\text { (Body) }\end{array}$ & 1. Neck & 2. Trunk & 3. Leg & - & & & & & \\
\hline \multirow{2}{*}{24} & \multirow{2}{*}{169} & \multirow{2}{*}{99.5} & \multirow{2}{*}{30} & $\mathrm{~A}$ & 2 & 1 & 2 & 2 & 3 & 0 & 1 & 4 & \multirow{2}{*}{3} \\
\hline & & & & B & 2 & 2 & 1 & - & 2 & 0 & 1 & 3 & \\
\hline 25 & 171 & 101 & 305 & $\mathrm{~A}$ & 2 & 1 & 2 & 2 & 3 & 0 & 1 & 4 & 4 \\
\hline 25 & 171 & 101 & $50 . J$ & $\mathrm{~B}$ & 3 & 2 & 1 & - & 3 & 0 & 1 & 4 & 4 \\
\hline & & & & A & 2 & 1 & 2 & 2 & 3 & 0 & 1 & 4 & \\
\hline 26 & $1 / 1$ & 101 & 30.5 & B & 3 & 2 & 1 & - & 3 & 0 & 1 & 4 & 4 \\
\hline 27 & 172 & 102 & 305 & $\mathrm{~A}$ & 2 & 1 & 2 & 2 & 3 & 0 & 1 & 4 & \\
\hline 21 & $1 / 2$ & 102 & 30.5 & B & 2 & 3 & 1 & - & 4 & 0 & 1 & 5 & 5 \\
\hline & & 1005 & 30 & $\mathrm{~A}$ & 2 & 1 & 3 & 2 & 4 & 0 & 1 & 5 & \\
\hline 28 & $1 / 2$ & 102.5 & 30 & B & 3 & 2 & 1 & - & 3 & 0 & 1 & 4 & 5 \\
\hline 20 & 170 & 102 & 30 & A & 2 & 1 & 3 & 2 & 4 & 0 & 1 & 5 & 5 \\
\hline 29 & $1 / 0$ & 102 & 30 & $\mathrm{~B}$ & 3 & 2 & 1 & - & 3 & 0 & 1 & 4 & 5 \\
\hline & & & & A & 2 & 1 & 2 & 2 & 3 & 0 & 1 & 4 & 3 \\
\hline 30 & $1 / 5$ & 104 & 30.5 & B & 2 & 2 & 1 & - & 2 & 0 & 1 & 3 & 3 \\
\hline & & & & $\mathrm{A}$ & 2 & 1 & 2 & 2 & 3 & 0 & 1 & 4 & 4 \\
\hline 31 & $1 / 6$ & 104.5 & 30.5 & $\mathrm{~B}$ & 3 & 2 & 1 & - & 3 & 0 & 1 & 4 & 4 \\
\hline 32 & 168 & 005 & 30 & $\mathrm{~A}$ & 2 & 1 & 2 & 2 & 3 & 0 & 1 & 4 & 5 \\
\hline 32 & 108 & 99.5 & 30 & B & 2 & 3 & 1 & - & 4 & 0 & 1 & 5 & 5 \\
\hline 33 & 160 & 1005 & 30 & $\mathrm{~A}$ & 2 & 1 & 3 & 2 & 4 & 0 & 1 & 5 & 6 \\
\hline 33 & 169 & 100.5 & 30 & B & 2 & 3 & 1 & - & 4 & 0 & 1 & 5 & 0 \\
\hline & & & & $\mathrm{A}$ & 2 & 1 & 3 & 2 & 4 & 0 & 1 & 5 & 4 \\
\hline 34 & $1 / 2$ & 102 & 30 & B & 2 & 2 & 1 & - & 2 & 0 & 1 & 3 & 4 \\
\hline 35 & 160 & 101 & 30 & $\mathrm{~A}$ & 2 & 1 & 2 & 2 & 3 & 0 & 1 & 4 & 3 \\
\hline 35 & 169 & 101 & 30 & B & 2 & 2 & 1 & - & 2 & 0 & 1 & 3 & 3 \\
\hline & & & & A & 1 & 1 & 1 & 2 & 2 & 0 & 1 & 3 & 4 \\
\hline 36 & $1 / 2$ & 103 & 30.5 & B & 3 & 2 & 1 & - & 3 & 0 & 1 & 4 & 4 \\
\hline 37 & 174 & 104 & 305 & A & 1 & 1 & 1 & 2 & 2 & 0 & 1 & 3 & 3 \\
\hline 31 & $1 / 4$ & 104 & 30.5 & B & 2 & 2 & 1 & - & 2 & 0 & 1 & 3 & 3 \\
\hline & & & & $\mathrm{A}$ & 2 & 1 & 2 & 2 & 3 & 0 & 1 & 4 & 4 \\
\hline 38 & 169 & 100 & 30 & B & 3 & 2 & 1 & - & 3 & 0 & 1 & 4 & 4 \\
\hline 30 & 157 & 605 & 30 & $\mathrm{~A}$ & 2 & 1 & 2 & 2 & 3 & 0 & 1 & 4 & 4 \\
\hline 39 & 151 & 09.5 & 30 & B & 3 & 2 & 1 & - & 3 & 0 & 1 & 4 & 4 \\
\hline 10 & 155 & 06 & 30 & $\mathrm{~A}$ & 1 & 1 & 1 & 2 & 2 & 0 & 1 & 3 & 3 \\
\hline 40 & 155 & 96 & 30 & B & 2 & 2 & 1 & - & 2 & 0 & 1 & 3 & 3 \\
\hline 41 & 170 & 102 & 30 & $\mathrm{~A}$ & 2 & 1 & 2 & 2 & 3 & 0 & 1 & 4 & 5 \\
\hline 41 & $1 / 0$ & 102 & 30 & B & 2 & 3 & 1 & - & 4 & 0 & 1 & 5 & 5 \\
\hline 42 & 150 & 05 & 30 & $\mathrm{~A}$ & 1 & 1 & 1 & 2 & 2 & 0 & 1 & 3 & 3 \\
\hline 42 & 150 & 95 & 30 & B & 2 & 2 & 1 & - & 2 & 0 & 1 & 3 & 3 \\
\hline 13 & 161 & 07 & 30 & $\mathrm{~A}$ & 2 & 1 & 2 & 2 & 3 & 0 & 1 & 4 & 3 \\
\hline 43 & 161 & 97 & 30 & B & 2 & 2 & 1 & - & 2 & 0 & 1 & 3 & 3 \\
\hline & & & & $\mathrm{A}$ & 2 & 1 & 3 & 2 & 4 & 0 & 1 & 5 & 6 \\
\hline 44 & 165 & 97.5 & 30 & B & 2 & 3 & 1 & - & 4 & 0 & 1 & 5 & 6 \\
\hline 15 & 168 & 08 & & $\mathrm{~A}$ & 2 & 1 & 2 & 2 & 3 & 0 & 1 & 4 & 3 \\
\hline 45 & 168 & 98 & 30 & B & 2 & 2 & 1 & - & 2 & 0 & 1 & 3 & 3 \\
\hline 46 & 165 & 075 & 30 & A & 1 & 1 & 1 & 2 & 2 & 0 & 1 & 3 & 3 \\
\hline 46 & 163 & 91.5 & 30 & B & 2 & 2 & 1 & - & 2 & 0 & 1 & 3 & 3 \\
\hline 17 & 166 & 00 & 30 & $\mathrm{~A}$ & 1 & 1 & 1 & 2 & 2 & 0 & 1 & 3 & 3 \\
\hline $4 /$ & 166 & 98 & 30 & B & 2 & 2 & 1 & - & 2 & 0 & 1 & 3 & 3 \\
\hline & & & & $\mathrm{A}$ & 1 & 1 & 1 & 2 & 2 & 0 & 1 & 3 & 4 \\
\hline 48 & 167 & 97.5 & 30 & B & 3 & 2 & 1 & - & 3 & 0 & 1 & 4 & 4 \\
\hline 40 & 150 & 065 & 30 & $\mathrm{~A}$ & 1 & 1 & 1 & 2 & 2 & 0 & 1 & 3 & 3 \\
\hline 49 & 159 & 90.5 & 30 & B & 2 & 2 & 1 & - & 2 & 0 & 1 & 3 & 3 \\
\hline & & & & A & 2 & 1 & 2 & 2 & 3 & 0 & 1 & 4 & 4 \\
\hline 50 & 167 & 97.5 & 30 & B & 3 & 2 & 1 & - & 3 & 0 & 1 & 4 & 4 \\
\hline
\end{tabular}


Further analysis of RULA result by user's height is shown in the following table.

Table 8. RULA Result Analysis by Height

\begin{tabular}{|c|c|c|c|c|}
\hline \multirow{2}{*}{ Height } & \multicolumn{2}{|c|}{ Dispenser type B } & \multicolumn{2}{c|}{ Dispenser type C } \\
\cline { 2 - 5 } & Quantity & Average Score & Quantity & Average Score \\
\hline$<167 \mathrm{~cm}$ & $15 \mathrm{resp}$ & 3.6 & $9 \mathrm{resp}$ & 3.5 \\
\hline$\geq 167 \mathrm{~cm}$ & $15 \mathrm{resp}$ & 4.1 & $11 \mathrm{resp}$ & 4.0 \\
\hline
\end{tabular}

Average RULA score at level 3.85 (later rounded up to 4) could mean that the design of existing water dispenser especially on water tap height has not been comfortable for respected user who has height more than $167 \mathrm{~cm}$. Bending body posture may cause further back pain or waist / buttock pain for certain user. So does the neck which bended more than $10^{\circ}$ may cause preliminary injury. Furthermore, RULA score 4 can be classified into Action Level 2 which means the body posture could present some risk of injury. Therefore, the change on the water dispenser may be needed in the near future to ensure an ideal user's body posture during the operation.

\subsection{Ergonomic Water Dispenser Improvement Recommendation}

According to RULA method, group B has bigger influence in defining the final RULA score rather than group A. Therefore, this research has been approaching on how to minimize the score in group B as a priority then followed by minimizing the score in group A. The expected posture has been set up as ideal as possible, then expected RULA score can be settled accordingly. The expected body posture as shown in the following figure.

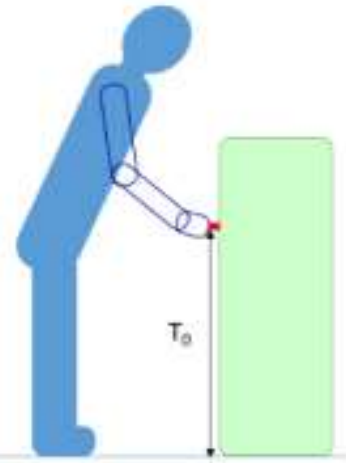

Existing Body Posture

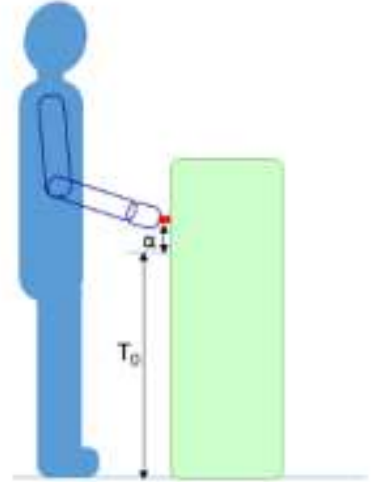

Expected Body Posture

Figure 5. Expected Body Posture on Improved Water Dispenser

The expected body posture in the figure above then deployed into group B and group A below to achieve improved RULA score at least level 2 which means minimum risk and safe to implement.

Table 9. Group B : Posture Setup and Expected Score

\begin{tabular}{|c|c|c|c|c|c|c|}
\hline & Neck & Trunk & Leg & $\begin{array}{c}\text { Activity } \\
\text { Score }\end{array}$ & Load Score & $\begin{array}{c}\text { Expected } \\
\text { Score B }\end{array}$ \\
\hline Position & $<10^{0}$ & $90^{0}$ & Normal & Static & $<2 \mathrm{~kg}$ & \\
\hline Value & 1 & 1 & 1 & 1 & 0 & 2 \\
\hline Score & \multicolumn{7}{|c|}{1} & 1 & 0 \\
\hline
\end{tabular}

Table 10. Group A : Posture Setup and Expected Score

\begin{tabular}{|c|c|c|c|c|c|c|c|}
\hline & $\begin{array}{l}\text { Upper } \\
\text { Arm }\end{array}$ & $\begin{array}{l}\text { Lower } \\
\text { Arm }\end{array}$ & Wrist & Wrist Twist & $\begin{array}{c}\text { Activity } \\
\text { Score }\end{array}$ & $\begin{array}{l}\text { Load } \\
\text { Score }\end{array}$ & $\begin{array}{c}\text { Expected } \\
\text { Score A }\end{array}$ \\
\hline Position & $<20^{0}$ & $60^{0}$ & Neutral & Center Position & Static & $<2 \mathrm{~kg}$ & \\
\hline Value & 1 & 1 & 1 & 1 & \multirow{2}{*}{1} & \multirow{2}{*}{0} & \multirow{2}{*}{2} \\
\hline Score & & & 1 & & & & \\
\hline
\end{tabular}


Based on expected score A 2 and expected score B 2 above, the expected final RULA score is 2. It is believed that the score could represent improved body posture when using water dispenser, there are upright back position, minimum angle in the neck area as well as improving angle around arm and hand area. Those body posture improvement required the change on water tap height level which had to be adjusted at $\alpha$ centimeter. By using trigonometric approach, $\alpha$ value measured through this following algorithm:

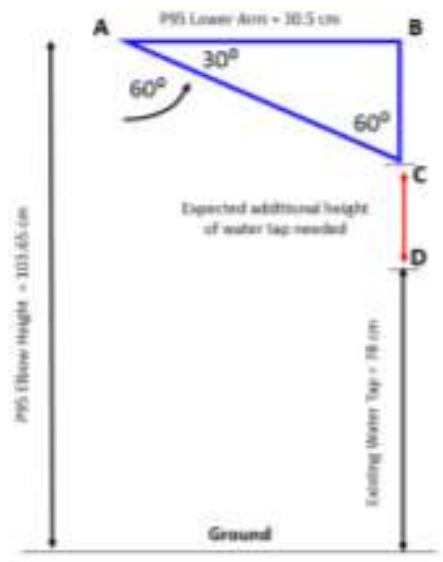

$$
\begin{aligned}
\frac{\mathrm{BC}}{\operatorname{Sin} 30^{\circ}} & =\frac{\mathrm{A} \mathrm{B}}{\operatorname{Sin} 60^{\circ}} \\
\mathrm{BC} & =\frac{30.5 \times 0.5}{0.86624}=17.60 \mathrm{~cm}
\end{aligned}
$$

Then, water tap height adjustment measured as follows:

$$
\begin{aligned}
& \alpha=\text { P95 Elbow Height }- \text { BC }- \text { Existing Water Tap Height } \\
& \alpha=103.55-17.60-80=5.95 \mathrm{~cm} \approx 6 \mathrm{~cm}
\end{aligned}
$$

The proposed water tap height level to achieve RULA score 2 is $86 \mathrm{~cm}$.

Figure 6. New Water Tap Height Level

Instead of adjusting water tap height level $6 \mathrm{~cm}$ higher, it is believed that modifying water tap valve has significantly contributed to achieve RULA score 2. The approach used to settle this task is on how to minimize second hand role to push or to press the existing water tap valve or button. The comparison and recommendation are shown in the table below (Scale of 5) :

Table 11. Water Tap Valve Mechanism Alternatives

\begin{tabular}{|c|c|c|c|}
\hline Criteria & Option 1 & Option 2 & Option 3 \\
\hline Description & Push Valve (Type A) & Smart Button : Start - Stop & Voice Command Sensor \\
\hline Conformance & 2 & 4 & 5 \\
\hline Reliability & 4 & 4 & 4 \\
\hline Serviceability & 4 & 4 & 3 \\
\hline Cost & 4 & 4 & $\mathbf{1 5}$ \\
\hline Total Value & $\mathbf{1 4}$ & $\mathbf{1 6}$ & 3 \\
\hline
\end{tabular}

Refer to comparison above, water tap with smart 'start - stop' button mechanism could deliver proper comfort for the user. One hand to grab water media and another could control water flow intermittently, at favorable cost.

\section{CONCLUSION}

Ergonomic water dispenser improvement has been proposed. Uncomfortable (bending) body posture when using water dispenser has been thoroughly measured and analyzed. The existing water dispenser is uncomfortable for those who has body height above $166 \mathrm{~cm}$. The RULA method to assess body posture on upper limb has been completely applied. Body posture in group B has played a significant role for RULA score rather than in group A. Several simulations have been performed. Water tap height level has been proposed to adjust around $6 \mathrm{~cm}$ higher than the existing level. Furthermore, water tap valve type has also been proposed to adopt smart 'start and stop' button mechanism to enhance ergonomic level of the hand.

\section{ACKNOWLEDGMENT}

The authors wishes to express the sincere thanks to the Center of Research of Universitas Mercu Buana for supporting and funding this research (Agreement No 02-5/897/B-SPK/IV/2020) 


\section{REFERENCES}

1. Anizar dkk (2018), Rancangan Fasilitas Kerja Ergonomis Pada Stasiun Pemarutan Tepung Tapioka, Seminar dan Konferensi Nasional IDEC (ISSN : 2579-6429).

2. Djiono, Yongly Kusnandar, et all (2013), Working Posture Analysis And Design Using RULA (Rapid Upper Limb Assessment) Method In Production Process at PT. Indana Paint, Jurnal Ilmiah Teknik Industri, Vol. 12, No.2,Desember2013 (ISSN 1412-6869)

3. McAtamney, L. and Corlett, E.N. (1993). "RULA -: A Survey Method for Investigation of Work related Upper Limb Disorders. Applied Ergonomics.

4. Nurmianto, Eko. (2004). Ergonomi, Konsep Dasar dan Aplikasinya, Edisi 2, Guna Widya, Surabaya.

5. Kottler, Philip (2010). Manajemen Pemasaran Jilid 2 ; Edisi 13, Erlangga, Jakarta.

6. Irianto, Djoko Pekik (2017). Pedoman Gizi Lengkap Keluarga dan Olahragawan, Edisi Revisi, Penerbit Andi, Yogyakarta.

7. Shabila, Anjani et all (2013), Design of Ergonomis Stool (Dingklik) for Batik Crafters, International Journal of Technology (ISSN 2086-9614).

8. Sokhibi, Akhmad (2017), Perancangan Kursi Ergonomis Untuk Memperbaiki Posisi Kerja Pada Proses Packaging Jenang Kudus, Jurnal Rekayasa Sistem Industri (ISSN : 2477-2089).

9. Sutalaksana, Iftikar (2006). Teknik Perancangan Sistem Kerja ; Edisi Kedua, ITB Bandung.

10. Sugiyono (2017). Statistika Untuk Penelitian. Alfabeta Bandung.

11. Suhardi, Bambang (2015). Perancangan Sistem Kerja ; Cetakan 1, UPT UNS Press Surakarta.

12. Torik (2015), Analisa Postur Dengan Metode RULA untuk Kerja Administrasi, Sinergi (ISSN: 1410-2331)

13. Ulrich, Karl T. dan Eppinger, Steven D. ; Perancangan dan Pengembangan Produk; Edisi Pertama - Jakarta ; 2001, Salemba Teknika, Jakarta

14. Yusuf, M. et all (2016), The Improvement of Work Posture Using RULA (Rapid Upper Limb Assessment) Analysis to Decrease Subjectie Disorders of Strawberry Farmers in Bali, International Research Journal of Engineering, IT \& Scientific Research (ISSN 2454-2261) 